\title{
Review of: "Comprehensive characterization of 536 patient-derived xenograft models prioritizes candidatesfor targeted treatment"
}

Liron Grossmann, John Maris

Potential competing interests: The author(s) declared that no potential competing interests exist.

Review by Liron Grossmann and John M. Maris

The development of new anticancer therapies has been challenged by the paucity of preclinical models that reliably recapitulate the response to therapy in human patients. Specifically, cell lines and cell-line derived xenografts (CDXs), which have been used to test drugs since the 1970s, lack predictive value for clinical trials and most newly developed agents fail to demonstrate clinical efficacy. This is partially explained by genetic and metabolic changes that cell lines undergo with time to adjust for the in vitro environment, which significantly differs from the in situ milieu. In addition, most cell lines and hence CDXs represent a homogenous population of cells and therefore lack the inherent intra-tumoral heterogeneity that characterizes their progenitor human tumors. Finally, cell-line derived models do not take into account the complex interaction that cancer cells have with their microenvironment, which has long been recognized to affect response to therapy.

Patient derived xenografts (PDXs), which are generated by directly implanting human tumor biopsy material into an immunodeficient mouse, have been shown to be more reliable preclinical models (1). Indeed, several studies have demonstrated a remarkable correlation between therapy response in patients and their corresponding PDXs (2), (3). As such, PDXs have been utilized in to prioritize candidate drugs for clinical trial, assess response to therapy, search for novel biomarkers and integrate them in co-clinical trials.

To accelerate translational research using PDX models, datasets and to promote collaboration, several programs were established to enable the development of large scale pre-clinical testing of targeted agents in both adult and pediatric PDX models. These include the NIH-NCI PDX Development and Trial Centers Research Network (PDXNet), the the EurpOPDX, the NIH-NCI Patient-Derived Models Repository (PDMR) and the Pediatric Preclinical Testing Consortium (PPTC, now known at PIVOT for pediatric in vivo testing program). The resulting large repositories of PDX models provide the research community with information about the PDX models, DNA and RNA sequencing data and associated clinical data. Our group has recently characterized 261 pediatric cancer PDX models and showed that PDX models recapitulate the genetic 
landscape of childhood cancers (4).

In a recent Nature Communications publication, Sun and colleagues performed a comprehensive genomic characterization of 536 PDX models from 511 patients across 25 adult cancer histologies. Using samples from the NIH-NCI PDXNet and PDMR initiatives, the authors analyzed the mutational landscape, copy number aberrations, cis-and trans-mutational statuses, define transcriptional groups, assess for clonal evolution in PDX models with multiple passages and identify PDX models that meet the NCl-MATCH study eligibility criteria. This study resulted in several important findings.

As expected by their high tumor purity, PDXs demonstrate a higher variant allele frequency (VAF) than the corresponding human progenitors. By comparing their mutational similarity, the authors report significant concordance between the PDXs and the human tumors in most types of cancer, underscoring the use of the PDX models in reliably studying and targeting genetic alterations in humans. Interestingly, the similarity score in a few types of cancers ( $10 \%$ of cancers) was markedly decreased with multiple mutations in human tumors not represented in the PDXs, suggesting a subclonal selection in the PDX generation process. This is an important observation that should be taken into account when testing new drugs in those specific PDX models. In addition, the authors also compare the intra- and inter-mutational similarity among different PDX models from the same patients and find higher intra-mutational than intermutational similarity supporting the notion of intra-tumoral heterogeneity within patients. This is further supported by copy number alterations (CNA) analysis demonstrating clonal evolution between different PDX models from the same patients. Importantly, while different PDX passages showed high mutational similarity, CNA changes were observed across passages from the same model. This suggests clonal evolution within the same PDX model over time, a finding that needs to be taken into account as PDX models that evolve over passage and perhaps diverge their human progenitors.

To determine the effect of key driver mutations, the authors performed cis- and trans-analyses using mRNA expression profiles. As expected, mutated tumor suppressor genes and oncogenes resulted in their downregulated and upregulated expression, respectively. Furthermore, trans-gene expression analyses revealed that several targetable genes that are co-expressed with mutated tumor suppressors and oncogenes. Mutations in ARIDIA, SOX9and RB1 correlated with upregulation of cell cycle genes CDK2, CDK4, CDKN1A, and CCNE2 in several types of cancers. The findings of such analyses can lead to the design of rational pre-clinical trials, for example CDK inhibitors targeting associated mutations in select PDX models.

Small molecules inhibitors have also been used to target fusion partners in cancers harboring fusion driver events. To test the effect of such inhibition on downstream pathways of the fusion partners, the authors compared the expression of key pathways genes between fusion positive and wild type PDXs. They found 
that FGFR3-TACC3 fusion in head and neck cancer PDXs was associated with upregulation of genes in the RAS-MAPK, JAK-STAT and PIK3-AKT, which are important for cell proliferation and survival. This finding suggests that clinically available FGFR inhibitors may be used to target pathways that are downstream to the fusion involving FGFR and establish a blueprint for studying other fusion events and their downstream gene expression patterns.

In summary, this study provides the translational research community with a useful resource for identifying PDXs that can serve as reliable pre-clinical models to prioritize anti-cancer drug candidates. While several observations, especially those associating mutation/fusion events with targetable pathways, require further functional validations, this work underscores the importance of comprehensively comparing PDXs and their human parental tumors to prioritize candidates for targeted therapy and the authors should be lauded for making these data freely available to the research community. Public-private partnerships will be required to most effectively leverage this and other such resources for efficient preclinical development of cancer therapeutics.

1. Hidalgo M, Amant F, Biankin AV, Budinská E, Byrne AT, Caldas C, et al. Patient-derived xenograft models: an emerging platform for translational cancer research. Cancer Discov. 2014;4(9):998-1013.

2. Tentler JJ, Tan AC, Weekes CD, Jimeno A, Leong S, Pitts TM, et al. Patient-derived tumour xenografts as models for oncology drug development. Nat Rev Clin Oncol. 2012;9(6):338-50.

3. Ice RJ, Chen M, Sidorov M, Le Ho T, Woo RWL, Rodriguez-Brotons A, et al. Drug responses are conserved across patient-derived xenograft models of melanoma leading to identification of novel drug combination therapies. Br J Cancer. 2020;122(5):648-57.

4. Rokita JL, Rathi KS, Cardenas MF, Upton KA, Jayaseelan J, Cross KL, et al. Genomic Profiling of Childhood Tumor Patient-Derived Xenograft Models to Enable Rational Clinical Trial Design. Cell Rep. 2019;29(6):1675-89.e9. 